\title{
Le « regard philologique » de Warburg
}

\section{Adi Efal}

\section{OpenEdition}

\section{Journals}

Édition électronique

URL : http://journals.openedition.org/imagesrevues/2853

DOI : 10.4000/imagesrevues.2853

ISSN : 1778-3801

\section{Éditeur :}

Centre d'Histoire et Théorie des Arts, Groupe d'Anthropologie Historique de l'Occident Médiéval, Laboratoire d'Anthropologie Sociale, UMR 8210 Anthropologie et Histoire des Mondes Antiques

\section{Référence électronique}

Adi Efal, « Le « regard philologique » de Warburg », Images Re-vues [En ligne], Hors-série 4 | 2013, mis en ligne le 30 janvier 2013, consulté le 31 janvier 2021. URL : http://journals.openedition.org/ imagesrevues/2853; DOI : https://doi.org/10.4000/imagesrevues.2853

Ce document a été généré automatiquement le 31 janvier 2021.

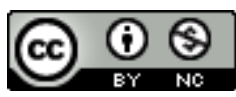

Images Re-vues est mise à disposition selon les termes de la Licence Creative Commons Attribution -

Pas d'Utilisation Commerciale 4.0 International. 


\title{
Le « regard philologique » de Warburg
}

\author{
Adi Efal
}

1 La contribution suivante ${ }^{1}$ 'sattache à présenter la conception warburgienne de l'histoire de l'art dans sa logique philologique. À nos yeux, l'histoire de l'art de Warburg, pratique proprement iconologique, a un fondement philologique. Une telle interprétation pose que le travail de Warburg a la même motivation que celui de Panofsky. C'est

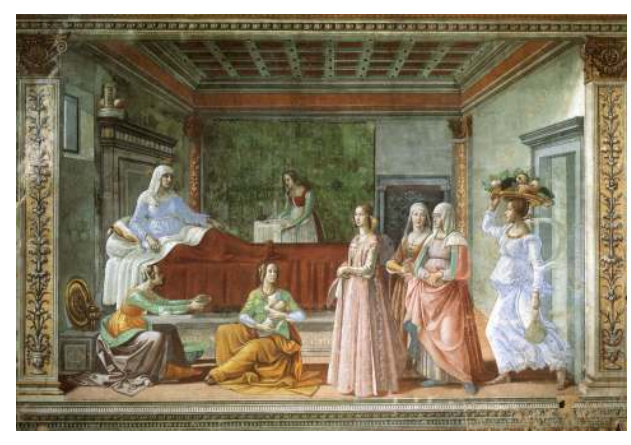
pourquoi, la restitution d'une impulsion philologique dans l'historiographie de l'histoire de l'art pourrait s'avérer un critère utile pour définir le lieu de notre discipline dans « l'atlas » des sciences humaines.

2 La bibliothèque de la science de la culture de Warburg à Hambourg fut en elle-même une sorte d'expérimentation philologique. ${ }^{2}$ L'idée de constituer une collection fonctionnant comme un réservoir de représentations, comme un «moteur de données » sur l'Antiquité classique et sa survivance dans la culture occidentale pendant des siècles, de même que la division dynamique des sections de la bibliothèque selon les motifs et les questions qui ont hanté Warburg toute sa vie, ${ }^{3}$ manifestent clairement une aspiration de type philologique à adhérer à la vie de l'Antiquité, à la préserver au moyen de la diagnose des traces, des indications de durée et de transformation des motifs. Les recherches de Warburg sur la transformation des atomes picturaux que sont les formules de pathos, combinaisons de signification et de forme, son attention bien connue aux détails et aux nuances de l'œuvre d'art, mais aussi son approche critique de la transfiguration du monde ancien, héritée de la tradition humaniste, tout cela atteste une orientation philologique évidente. ${ }^{4}$

3 Dans un essai intitulé "Warburg et la science sans nom ", daté de $1975,{ }^{5}$ Giorgio Agamben a tenté de définir la nature singulière, difficilement nommable, de l'histoire 
de l'art de Warburg. D'une certaine façon, je voudrais établir que cette «science warburgienne sans nom " peut être définie comme une philologie figurale. Dans un premier temps, j'envisagerai l'aspect philologique du travail de Warburg. Dans un second temps, je considèrerai succinctement la nature figurale de cette philologie.

Les données biographiques révèlent que bien des philologues ont croisé le chemin de Warburg. J'en retiendrai seulement quelques-uns.

5 Comme l'indique Agamben, ${ }^{6}$ la prise en compte de la philologie mythologique d'Hermann Usener, fort connue à l'époque, est essentielle à la compréhension de la pensée warburgienne. Durant ses années d'étude à Bonn, Warburg assista aux conférences d'Usener. Dans une lettre écrite à ses parents, quelques semaines après son arrivée dans cette ville, il notait :

En fait de philosophie le cours de Usener me suffit. Quant au professeur Justi, qui est le numéro un en histoire de l'art, j'irai l'écouter le semestre prochain, après m'être penché de plus près sur des travaux proprement philologiques. ${ }^{7}$

6 Cette citation nous apprend que Warburg considérait le savoir philologique comme un préalable aux études d'histoire de l'art. Par ailleurs, nous comprenons qu'il envisageait l'approche d'Usener comme philosophique et donc, qu'à ses yeux, il y avait une sorte de continuité entre philosophie et philologie, continuité cachant toutefois aussi, comme nous le verrons, une césure décisive. Usener est connu pour sa conception de la formation des noms divins qui, selon lui, est le résultat d'une réaction physicopsychologique primitive vis-à-vis des puissances naturelles. C'est cette conception qui a été adoptée par Warburg dans sa formule de pathos. ${ }^{8}$ Mais Usener a été également considéré comme un savant attaché à la tradition historique et culturelle de la philologie allemande du XIX siècle. Pour lui, la philologie est la condition nécessaire à toute activité historique. ${ }^{9}$

7 L'autre professeur mentionné par Warburg dans la citation ci-dessus, Carl Justi, a pour sa part pratiqué une histoire de l'art essentiellement biographique, reprenant le modèle vasarien de l'histoire des héros (« Heldengeschichte »), des individus hors du commun et créateurs, envisagés comme les piliers d'une tradition canonique. ${ }^{10} \mathrm{En} 1898$, notamment, il a publié une étude approfondie sur Johann Joachim Winckelmann, figure dotée elle-même d'une certaine pertinence sur le plan philologique. De formation philologique classique, Justi avait fait sa thèse doctorale sur les éléments esthétiques de la philosophie de Platon, en combinant des approches hégéliennes et néo-kantiennes. ${ }^{11}$ De fait, il semble que l'attitude critique de Warburg contre la tendance idéalisante en histoire de l'art, ait été en partie nourrie par son contact avec Justi.

8 Autour de 1900, la pratique philologique était divisée: d'un côté, il y avait une philologie soutenant la primauté de l'idéal gréco-romain, de l'autre, se trouvait une " autre " philologie, cherchant à redéfinir cet idéal même et mettre en évidence sa complexe formation. Cet autre courant était dominé par l'école dite de "Neuphilologie » qui avait commencé de mener des recherches « morphologiques » sur des époques nonclassiques, c'est-à-dire essentiellement sur le monde médiéval..$^{12}$ Parmi les représentants de ce courant, Anna Guillemin retient Karl Voßler et d'Hermann Osthoff comme déterminants pour comprendre les orientations de Warburg. ${ }^{13}$

9 Karl Voßler pratiquait une philologie idéaliste et vitaliste, profondément inspirée par la philosophie de Benedetto Croce, qui s'opposait aux tendances ultra-positivistes de la science linguistique. L'on sait que Warburg écrivit à Voßler en 1927 pour l'inviter à donner une conférence dans sa bibliothèque ${ }^{14}$ En dépit des accents ultra-idéalistes de 
cette école croceiste de la philologie, ${ }^{15}$ je considère qu'elle permet d'éclairer le travail de Warburg. ${ }^{16}$ Mais il faut nous garder de faire de Warburg un penseur exclusivement idéaliste, ce qui occulterait le caractère précis de son rapport à la réalité archaïque. Pour la même raison, on ne saurait le rapprocher trop d'Ernst Curtius, le grand philologue de la culture romane, pourtant proche des intérêts de la bibliothèque Warburg et dont le travail manifeste un identique caractère idéaliste. ${ }^{17}$ Pour Warburg, en effet, ce n'est pas l'esprit qui fonde la production artistique, c'est surtout l'organisme vivant, le corps "pathique ", élastique et plastique qui survit en elle.

Quant à Osthoff, il était le représentant d'un autre genre de philologie que nous pouvons nommer « positiviste ». Warburg avait annoté une des conférences donnée par Osthoff à Heidelberg en 1899 sur la notion de "supplétion ». ${ }^{18}$ La supplétion linguistique désigne l'ensemble des formes prises par un même lemme qui, dans sa flexion, fait intervenir différents radicaux, produits d'irrégularités et de modifications. Indiscutablement, ce processus «supplétif» est proche de la dynamique des formules de pathos de Warburg. En somme, il semble que la direction morphologique soit plus pertinente pour saisir ses intuitions que l'orientation explicitement idéaliste. Les développements ultérieurs le prouvent. Quelques années plus tard, en 1909, un autre groupe de même inclinaison morphologique,$^{19}$ basé à Heidelberg, fonda la revue Wörter und Sachen (Les mots et les choses (!)), qui élargit le champ d'application morphologicoétymologique aux recherches des instruments et des monuments. ${ }^{20}$ Une telle méthode, qui tente de suivre les nuances et les variations de formes dans leur déclinaison et leur usage, est assurément proche de celle de Warburg.

11 Mais c'est surtout et avant tout l'influence de Friedrich Nietzsche qui conféra aux recherches de Warburg un caractère philologique décisif, car, située elle aussi du côté d'une " autre philologie », elle place au cœur des choses la mise en question de l'idéal. ${ }^{21}$ L'attention portée à la composante nietzschéenne de la pensée de Warburg s'est jusqu'à maintenant focalisée sur la tendance vitaliste, voire dionysienne, des deux penseurs. Elle a été moins attentive au fait que Nietzsche, tout comme Warburg, s'est interrogé sur le statut de l'Antiquité et sur sa survivance dans la culture occidentale, et s'est penché sur son traitement par les sciences philologiques. ${ }^{22}$

12 Le texte Die Geburt der Tragödie de Nietzsche est justement le produit d'un dialogue difficile avec la philologie classique, et le philologue canonique de l'époque, Ulrich von Wilamowitz-Möllendorff, l'avait d'ailleurs critiqué avec grande férocité, comme si l'avenir entier de la philologie en eût été menacé. ${ }^{23}$ À une date proche de la publication de son texte sur la tragédie, Nietzsche avait rédigé le brouillon d'un essai intitulé Wir Philologen, ${ }^{24}$ dans lequel il affirmait sa vocation philologique. Toutefois, sa position visà-vis de la philologie académique y était très critique, car il revendiquait une pensée radicalement nouvelle de cette science qui, a ses yeux, n'avait pas encore vu le jour. Pour Nietzsche, il y avait obligation à évaluer le passé au regard des valeurs de son observateur, autrement dit des valeurs du philologue lui-même. À cette orientation radicale se rattache Warburg, ce qui ne l'empêche pas de témoigner un égal respect visà-vis de la philologie canonique de von Wilamowitz-Möllendorff. Pour preuve, le fait qu'il invita personnellement ce dernier à donner une conférence dans sa bibliothèque en $1924 .{ }^{25}$

13 Nietzsche, Usener et Voßler, tous trois partisans de l'«autre philologie », avaient chacun à sa manière pratiqué une philologie imprégnée de Lebensphilosophie. Tous s'essayaient à conférer une fondation vitale à la production des signes : dynamique 
dionysienne dans le cas de Nietzsche, mouvement des puissances naturelles dans celui d'Usener, enfin, création (Schöpfung) pour Voßler. Dans cette perspective vitaliste, nous pouvons aussi inclure Warburg, si ce n'est que pour lui, ce n'est pas la vie qui fonde le fait artistique, mais plutôt une organisation "pacifique", un dispositif créateur de formules frayant des traces mémorielles. ${ }^{26}$

Il est possible d'aborder cette tendance vitaliste du point de vue de l'épistémologie historique, à partir de l'interrogation sur les choses survivantes venant du passé. En ce sens, la philologie de Warburg peut être mise en rapport avec la crise historiciste qui a agité les fondements des sciences humaines tout au long du XIX ${ }^{e}$ siècle,${ }^{27}$ crise liée à la position ontologique du passé, à la question de sa réalité. Le passé est-il une chose, une chaîne d'événements ou encore un complexe de phénomènes? Le passé constitue-t-il une source vive pour la culture ou bien n'est-il qu'un reliquat à utiliser au gré des besoins de la logique contemporaine ? La réalité historique du passé est-elle relative aux nécessités et aux valeurs de l'historien ou bien les choses du passé -monuments et documents- opposent-elles quelque force de résistance au relativisme historique ?28

Durant le XIX ${ }^{e}$ siècle, la philologie tout autant que l'histoire de l'art ont bénéficié dans leur existence académique d'une position particulière leur permettant d'échapper en partie au relativisme historiciste. Et cela grâce à l'enracinement de leurs discours dans l'idée de la survivance et de la transmission de la tradition antique, dont le fondement était censé se situer en deçà des hasards des vicissitudes historiques. Aux yeux du philologue, l'Antiquité ne constitue pas seulement un point d'origine temporel: la strate classique produit des formules, des points d'appui, des critères plus ou moins stables qui peuvent faire office de "points d'Archimède ${ }^{29}$ pour le récit historique. En histoire de l'art, comme en philologie, les postulats de l'historicisme, c'est-à-dire la temporalisation de l'être et l'identification de l'existence humaine avec l'existence historique, n'ont pas été retenus comme des principes absolus. Pour une discipline comme pour l'autre, l'histoire n'est pas tout, elle ne couvre pas la surface entière de la réalité du passé sur lequel elles se penchent. À côté du flux temporel, il y a aussi des formules qui se répètent (en produisant des figures, comme nous allons le voir par la suite), des formules qui gardent leur cohérence dans l'histoire. Sur ce point, l'histoire de l'art et la philologie ont été solidaires. Pour elles, l'histoire n'est pas un devenir libre, pas davantage un processus strictement dialectique, mais elle est plutôt configurée par des prototypes et des formules..$^{30}$

16 La relation épistémique avec le passé, qui passe par le chemin de la survivance de l'Antiquité, prend place dans l'histoire de l'art warburgienne avec la notion de "formule de pathos ", atome pictural qui fixe une réalité dynamique extérieure. Mais quelle est la faculté apte à discerner les formules de pathos à l'œuvre dans l'histoire des arts? Je suggère que cette faculté est précisément ce que Warburg lui-même a nommé son « regard philologique».

17 L'anthologie récente des œuvres de Warburg dirigée par Sigrid Weigel, Martin Treml et Perdita Ladwig contient un fragment daté de 1900 qui porte sur la «Nymphe florentine $»^{31}$ de Ghirlandaio figurant sur les fresques de la chapelle Tornabuoni de l'église Santa Maria Novella de Florence, fragment que seule la biographie intellectuelle d'Ernst Gombrich avait jusqu'à maintenant signalée, et encore sous une forme incomplète. Dans ce texte, qui contient un échange de commentaires avec le philologue et historien de l'art Johannes Andréas Jolles, ${ }^{32}$ Warburg se réfère à son "regard philologique » (" philologischer Blick $\left.»^{33}\right)$. Certes, l'expression est seulement mentionnée 
en passant, mais pour notre propos, elle a une portée considérable. Comment la comprendre ? Comment un regard peut-il être " philologique»? Autrement dit, et l'on retrouve ici l'éternelle question, comment est-il possible d'établir une correspondance fidèle et réelle entre appréhension visuelle et formulation linguistique ? Derrière les interrogations de Ludwig Wittgenstein, Jacques Derrida, Louis Marin ou encore J.W.T. Mitchell, il est possible de découvrir une dimension philologique. C'est par la voie de l'inscription, voire de l'écriture, porteurs plastiques du langage, que la correspondance recherchée est trouvée. Or, la philologie est justement le terrain où l'écriture et sa réalité plastique se touchent et s'entrelacent. Elle est une mise-en-abyme de ce que nous cherchons. Si nous examinions les écrits des fondateurs de l'histoire de l'art, de Winckelmann à Panofsky, nous découvririons que les instruments philologiques ont servi dans la constitution de cette discipline hantée et aberrante. S'appuyer sur la philologie oblige toutefois à une infime (mais dramatique) modification de point de vue : ce n'est pas l'aesthesis du regard, donc l'image, qui se trouve confrontée au langage, mais plutôt l'agencement figural, c'est-à-dire la réalité plastique qui pousse, déplace et installe la réalité physique elle-même, toujours en mouvement, vers ou depuis une forme. ${ }^{34}$ Dans l'optique d'une telle approche, il n'y a pas de dichotomie entre langage et formes visibles, mais une complète continuité.

Nous allons analyser l'utilisation précise que Warburg fait de l'expression « regard philologique ». Avant toute chose, il nous faut examiner la totalité de la phrase dans laquelle elle fait son apparition et, pour cela, reprendre la traduction anglaise proposée par Gombrich en indiquant, en plusieurs endroits, les termes allemands correspondant : ${ }^{35}$

You (Jolles, l'ami de Warburg) feel prompted to follow her (i.e. la Nymphe) like a winged idea (geflügelten Idee) through all the spheres in a Platonic frenzy of love; I feel compelled (mich zwingt sie) to turn my philologician's gaze to the ground (Boden) from which she rose and to ask with surprise : "Is this strangely delicate plant really rooted in the sober Florentine sole (Erdboden) ?"

Gombrich a traduit « den philologischen Blick » par « My philologician's gaze », or, il semble qu'une traduction neutre (soit « le regard philologique » et non « mon regard philologique») aurait été plus en accord avec le texte original. Elle aurait donné à l'expression un tour un peu moins biographique et davantage conceptuel. En premier lieu, nous devons remarquer que Warburg lui-même a choisi l'adjectif philologique alors qu'il aurait pu lui en préférer d'autres et parler de " regard historique » ou encore de « regard herméneutique ». Il est donc tout à fait clair que Warburg avait identifié son attention comme dotée d'un caractère philologique. En deuxième lieu, il faut noter que Warburg a opposé son regard philologique au regard platonique, idéaliste, de son ami. ${ }^{36}$ Si le regard platonique, théorique, est attiré par l'érotisme "eidétique », le regard philologique, en revanche, tente de découvrir le «sol» (Erdboden) de l'image. La philologie cherche les racines, les principes initiaux des réalités poïétiques (c'est-à-dire produites). ${ }^{37}$ Le regard philologique voit donc par et à travers l'image; il la creuse. Ce regard essaye de dépasser l'aspect captivant de l'image comme de l'idée et tente de capturer le principe générateur, l'arché ( $\alpha \rho \chi \eta ́)$, qui se trouve à leur fondement. ${ }^{38}$

Le regard philologique n'est pas un regard indifférent, naïf ou passif. Ce n'est pas un regard correspondant à un point de vue naturel, pas plus qu'à une approche phénoménologique, esthétique, descriptive ou réflexive. En revanche, c'est un regard habité, lourd d'une tradition, donc imprégné d'histoire, un regard qui-a-déjà-vu, qui porte un passé, un «regard âgé », doté d'une puissance mnémonique. Le regard 
philologique est celui qui est supposé savoir quelque chose, a priori, de son objet ; c'est donc un regard plein de préjugés, chargé de lourdeurs, de questions, de motivations, d'inclinations et de dispositions.

21 Par conséquent, il faut envisager la possibilité que le regard du philologue ne soit pas celui qui produit les formules de pathos mais plutôt celui qui les reçoit. Si la formule de pathos est le résultat d'un regard virginal, non-préparé pour une rencontre avec une force extérieure, le regard philologique est, quant à lui, protégé contre les rencontres traumatisantes avec l'extérieur menaçant. Au moins est-il partiellement préparé pour la rencontre avec la séduction érotique de l'image et avec la nature extérieure. Il me semble que le regard philologique est le regard complémentaire du regard produisant la formule de pathos: si ce dernier conjure la dimension menaçante des puissances naturelles, le premier rouvre l'image pour re-mobiliser la formule conjurée. La formule de pathos accueille la réalité extérieure, alors que le regard philologique reconduit la chose picturale, appréhendée comme donnée, à son propre fonds et la ramène à ses propres causes. C'est pourquoi, il est nécessairement un regard synthétique qui s'appuie sur des homologies (selon lesquelles un caractère en commun signifie une même origine).

Pour décrire un processus similaire, Erwin Panofsky a utilisé le terme particulier d'intuition synthétique (synthetic intuition), ${ }^{39}$ par quoi il faut entendre la capacité à localiser une œuvre d'art spécifique dans l'histoire des symptômes culturels. Le regard philologique de Warburg peut être assimilé à cette intuition de type synthétique (notion qui d'ailleurs mériterait en elle-même une étude approfondie). Comme il ramène le tableau à son fonds, le regard philologique établit la nature seconde d'une réalité picturale. En utilisant un terme aristotélicien-thomiste, nous dirions qu'il perçoit l'habitus ( $\check{\varepsilon} \xi \zeta \zeta^{40}$ qui est porté par l'unité picturale mais n'est pas immédiatement aperçu par le spectateur. Ce sens philologique n'est pas révélé mais construit, dans la mesure où il est mis en œuvre à l'occasion de la lecture de documents ou de monuments différents, ce qui permet d'établir une série de répétitions typiques de formules.

Pour Warburg, la racine de l'image est double. D'un côté, il recherche les conditions matérielles, sociales et économiques qui président à la production d'une œuvre. Ainsi, la Nymphe est lue par lui comme une manifestation de la mentalité non religieuse des familles de banquiers de la Florence du $\mathrm{XV}^{\mathrm{e}}$ siècle. La seconde racine de l'image est la formule de pathos, le type archaïque, qui ressurgit à travers les âges, les cultures et les acteurs successifs. Ces deux racines peuvent être décrites comme matérielles: d'un côté, il s'agit des conditions matérielles de telle ou telle époque passée, de l'autre côté, on a affaire à une réalité plus archaïque, également matérielle, dans le sens où, pour reprendre les termes de Usener dans la perspective de Warburg, toute formule de pathos est l'enregistrement d'une rencontre archaïque avec la nature. La production porte donc en elle une dimension archaïque qu'il faut conjoindre à une dimension historique, résultat de transmissions, de contextes et de transferts.

Le regard philologique est décrit par Warburg comme la mise-en-acte d'un doute méthodique qui, tout en traitant avec scepticisme le pouvoir captivant de l'image et des idées, est animé par la certitude qu'il est possible de découvrir le double fondement de l'objet pictural. ${ }^{41} \mathrm{Ce}$ doute modéré est, semble-t-il, proche du doute cartésien: s'il résiste à l'aspect séduisant des images et des sensations, il reste cependant sous-tendu par une certitude. Or, la philologie regarde comme certitude fondamentale la réalité du 
passé produit par l'homme. C'est le factum de Vico, la chose fabriquée, qui donne cette certitude à la science philologique. ${ }^{42}$

L'objet de la reconstitution warburgienne est la formule de pathos, c'est pourquoi le regard du philologue est attiré par des images imprégnées d'un passé intensif codé. La tâche de ce dernier est de déterminer un lien entre un donné spécifique (l'objet pictural rencontré par le chercheur) et un prototype de cette même image (par exemple, la servante de la fresque de Ghirlandaio identifiée comme une nymphe). C'est au cœur même de la distance entre les deux que la philologie travaille. Le regard philologique, qui saisit cette distance, est un regard stéréoscopique qui identifie les deux racines d'une image donnée et les découvre synthétisées dans une nouvelle création distincte. Il est au service de la restitution d'un habitus déposé dans un objet pictural. Il est un processus de diagnostic qui commence par mettre en évidence, par isoler un détail de l'œuvre porteur d'une formule de pathos. L'enquête iconologique, à la manière d'une philologie figurale, essaye de repérer la façon dont la formule de pathos s'est transmise d'un âge à l'autre, d'une culture à l'autre pour finir par aboutir à l'objet pictural examiné. Cette lecture rend compte de la façon dont la dite formule ressurgit après s'être assoupie et être restée endormie, latente, dans le sein obscur de l'histoire.

La conception warburgienne de la formule de pathos ne peut être séparée de l'idée de mobilité. Aux yeux de Warburg, le mouvement inhérent à la formule de pathos a été rattaché à la qualité de réalisme. Le penseur qui a formulé le plus nettement l'équivalence entre la réalité et le mouvement, au moment même où Warburg lui-même élaborait ses théories, est Henri Bergson: "Il y a une réalité extérieure et pourtant donnée immédiatement à notre esprit [...] Cette réalité est la mobilité. $»^{43}$ La mobilité même, avec la formule qui la figure, est donc la réalité que le regard philologique perçoit.

Dans sa thèse de 1893 sur le Printemps et la Vénus de Botticelli, ${ }^{44}$ Warburg avait déjà examiné la manière dont la représentation picturale d'éléments mouvants et flottants était considérée par des critiques et des poètes florentins $\mathrm{du} \mathrm{XV}$ siècle comme porteuse de pouvoir et de validité réalistes. L'utilisation des formules de pathos dans l'art occidental est un instrument pour créer une impression réaliste et, en retour, c'est grâce à l'impression réaliste que la formule de pathos est dotée d'une efficacité rhétorique persuasive. Encore une fois, c'est Henri Bergson qui peut nous aider à comprendre que l'instrument mental associé à cette réalité mouvante soit la mémoire et non la représentation ou la compréhension. ${ }^{45}$

Le réalisme inhérent à la formule de pathos conserve et remémore la réalité du mouvement. Dès lors, cette dernière porte une dimension mnémonique et c'est précisément cette dimension dont s'occupe la rationalité philologique. Cette forme mouvante n'est pas une forme au sens platonicien de l'zĩ $\delta o \varsigma .{ }^{46}$ Pour que la forme soit en mouvement, il faut qu'elle ait d'abord été corps et lieu. Cet hylémorphisme concret a posteriori et non détachable est, selon moi, non plus «formel», mais proprement figural. ${ }^{47}$ Or, si l'on en croit le modèle figural de Gilles Deleuze, par exemple, l'hylè de la figure, ce sont les corps et les forces..$^{48}$

Les réflexions de Bergson et de Warburg prouvent exemplairement qu'autour de 1900, la " réalité » avait été pensée comme une entité essentiellement mnémonique ${ }^{49}$ et que le réalisme de la mémoire allait de pair avec la rationalité philologique. réalité du passé des choses fabriquées par l'homme : c'est le domaine de la production 
et de la génération, caractérisé par Aristote comme le champ poïtique de l'action humaine, qui forme son terrain d'exercice. ${ }^{50} \mathrm{En}$ ce sens, la philologie ne s'intéresse pas aux événements ni aux attitudes mentales, pas plus qu'aux idées ou concepts cachés dans les textes ou les œuvres d'art. Elle se confronte à la mobilité qui est celle des actes de production et de génération. ${ }^{51}$ Les réalités poïétiques sont considérées par elle comme des éléments de préservation, de conservation, et de la vie et de la survivance (Nachleben) de la vie. ${ }^{52}$ Dotés de cette fonction préservatrice, les objets philologiques sont envisagés comme orientés vers le futur, mais aussi vers le passé. Nécessairement, donc, ils établissent une tradition. La tâche du philologue est de "coller » aux formulations, aux mots et aux choses du passé. C'est ainsi qu'il entend l'appel de la formule de pathos. En ce sens, le corpus philologique appartient à ce que l'on peut nommer un univers humaniste ${ }^{53}$ puisqu'il opère des communications à travers les âges et les époques. Cette aptitude du regard philologique conduit tout droit à la tension que l'on trouve chez Warburg entre, d'une part, l'attention donnée aux dialectes particuliers, aux caractères locaux, aux détails distinctifs qu'il a tellement affectionnés et, d'autre part, l'histoire universelle dans laquelle ces dialectes ou ces détails prennent place, tension manifestée par l'architecture même de l'Atlas Mnémosyne.

31 Les formules de pathos sont des agents de transfiguration, de transmission, d'un médium à l'autre, d'un âge à l'autre, d'un corps à l'autre, d'un instrument à l'autre. Pour retracer une telle transfiguration, il faut reconstituer un ethos, une disposition ou une forme de comportement habituel, une tradition porteuse d'un conatus se manifestant dans la durée. Il revient au philologue d'esquisser de telles pistes généalogiques. Néanmoins, quand il établit une logique figurale, il ne se montre pas toujours fidèle à la réalité des événements historiques. Plus encore, dans ses formes les plus pointues, la philologie n'a presque rien à voir avec l'histoire..$^{54}$ Mais le regard philologique de Warburg n'est pas aussi radical : chez lui, l'histoire constitue toujours une considération déterminante.

De fait, nous voudrions suggérer que, chez lui, l'attention philologique à la réémergence des formules et la présentation synchronique d'une œuvre et de ses prototypes produisent à leur tour une figure. Les principes tirés du fragment sur la " Nymphe " mentionnés plus haut, l'enregistrement du mouvement, la manière dont les traits transmettent des formules d'une culture à l'autre, la façon dont une certaine organisation picturale incorpore un habitus et surtout, l'effort warburgien pour formuler adéquatement le sens des réalités poïétiques, sans prendre appui, pour ainsi dire, sur les ailes des idées, tout cela nous rapproche de la notion de figura examinée par Erich Auerbach dans un célèbre essai de $1938 .{ }^{55} \mathrm{Si}$ la formule de pathos indique le moment de fixation d'un habitus, c'est-à-dire le moment où une manière d'être se fixe dans une forme picturale (afin de maîtriser une réalité extérieure menaçante), la figure, quant à elle, incarne la répétition du même habitus dans une série d'œuvres séparées dans l'histoire. Dès lors, l'habitus conservé par une figure n'est pas une suite de conditions de possibilité, mais une manière d'être, un comportement d'acteurs humains.

Figure est un mot d'origine latine (figura) qui signifie, en son sens général, l'aspect extérieur des choses et qui, en un sens plus précis, désigne les dispositifs paralinguistiques grâce auxquels un discours gagne en force persuasive. ${ }^{56}$ Plus tard, dans le cadre de la préfiguration chrétienne, il s'agit surtout des diverses sortes de répétitions analogiques de portions de l'histoire humaine qui forment les atomes du registre 
figural. Dans les écrits théologiques chrétiens, les structures figurales sont constituées par les discours de la réalisation (Erfüllung) et par ceux de la " prophétie réelle.$^{57}$ Elles se construisent au minimum par le biais de deux réalités historiques, qui sont souvent des personnages, comme Adam, Josué, Jésus, Moïse ou Virgile, mais peuvent être aussi des événements. ${ }^{58}$ Le dynamisme figural associe ces deux réalités historiques l'une à l'autre. Ainsi, de même que Moïse a conduit le peuple d'Israël aux portes de la terre promise, de même le Sauveur conduit ses fidèles aux portes de la Jérusalem nouvelle. $\mathrm{Et}$, dans le texte sur la figure où il envisage de telles constructions typologiques, Auerbach examine finalement les figures qui apparaissent dans la Divine Comédie de Dante. Dans ce cas, la rationalité figurale s'appuie sur la réalité concrète de divers personnages, appartenant à des siècles éloignés dans le temps, mais qui se rencontrent dans les pages de l'ouvrage grâce au réseau constitué. Nous avons noté plus haut l'importance de l'argument réaliste dans la recherche de Warburg sur Botticelli. On comprend donc que ce qui se joue dans la formule de pathos comme dans la figure, c'est la crédibilité du signe, la certitude, toujours découverte a posteriori et par un mouvement rétrograde, qu'il y a quelque réalité contenue dans la chose.

Il faut préciser. Je ne veux pas dire que la formule de pathos de Warburg soit identique à la figure d'Auerbach. Les deux concepts sont évidemment bien distincts. Mais, dans le cadre du travail proposé ici, il me semble possible de concevoir la figure comme une structure conditionnant, pour le regard philologique, la détection d'une formule de pathos. Certes, Auerbach n'a pas travaillé à la bibliothèque Warburg à Hambourg; en revanche, il a envisagé la possibilité de visiter la nouvelle installation de la bibliothèque à Londres. ${ }^{59}$ En mentionnant Auerbach, nous voici reconduits à la question de la philologie idéaliste. Auerbach, en effet, avait soutenu son habilitation avec Karl Voßler, le philologue vitaliste croceiste évoqué plus haut et l'on sait qu'il avait obtenu le poste de professeur de Romanistique, comme successeur de Spitzer à Marburg, grâce à la recommandation de Voßler et de Croce. ${ }^{60}$ Pas davantage que Warburg, toutefois, Auerbach ne saurait être réellement considéré comme vitalo-idéaliste car c'est la réalité historique qui fonde sa vision philologique.

Nous sommes davantage habitués à souligner l'intérêt de Warburg pour le pathos et l'agitation plus qu'à réfléchir sur l'aspect éthique de son travail. Mais, en réalité, il y a un lien essentiel entre le pathos et l'ethos dans ses œuvres. ${ }^{61}$ C'est par la détection et la détermination des formules de pathos que le chercheur peut reconstituer un ethos pris dans la durée. La formule de pathos nous aide à remonter, à faire le chemin à rebours, à régresser sur l'échelle de la mémoire dite culturelle, elle nous invite à rouvrir l'image et à « déplier » l'éthos contenu pour récréer l'intuition synthétique du passé poïétique.

Avec l'agencement de la figure, la philologie, telle qu'elle est décrite dans les écrits d'Erich Auerbach, opère la présentation (Darstellung), la réalisation (Erfüllung), la mise en scène, la dramaturgie d'une tradition, au travers des âges, des cultures et des acteurs séparés par l'histoire. Ce genre de présentation est effectué dans les œuvres d'Erwin Panofsky, Leo Spitzer et Ernst Curtius, s'en tenir à quelques exemples..$^{62}$ L'Atlas Mnémosyne réalise lui aussi la représentation d'une tradition, celle de la transmission des formules de pathos qui_configurent l'histoire de la poïsis occidentale. L'ultime technique utilisée par Warburg consiste en l'occurrence à juxtaposer des fragments imagés, collés sur des planches séparées mais pouvant être rapprochées, en accord avec l'ordre rationnel de la bibliothèque même. Il ne s'agit pas exactement d'arbres généalogiques ni d'illustrations ordonnées par des arguments: les compositions de 
l'Atlas réalisent avant tout un espacement qui peut être effectivement conçu comme " espassément », c'est-à-dire comme mise-en-place du passé.

Dès lors, il ne suffit pas de dire qu'il existe des affinités profondes entre l'histoire de l'art warburgienne et la méthode philologique. Il faut conclure que le travail de Warburg mène sur la voie d'une philologie dont le fondement même est figural.

\section{NOTES}

1. Mes remerciements vont aux fondations Fritz Thyssen (2010-2012) et Gerda Henkel (2012-2013) sans le soutien desquelles cet article n'aurait pas vu le jour. Je remercie aussi de tout cœur Sabine Forero-Mendoza et Jonathan Touitou pour leur aide dans la mise au point de la version française de ce texte.

2. Voir Martin Jesinghausen-Lauster, «Die Warburg Bibliothek als Problem », Die Suche nach der symbolischen Form. Der Kreis um die Kulturwissenschaftliche Bibliothek Warburg, Baden-Baden, Verlag Valentin Koerner, 1985, p. 151-217.

3. Sur la bibliothèque Warburg à Hambourg voir Philippe-Alain Michaud, « Hambourg. La scène de l'histoire de l'art ", Aby Warburg et l'image en mouvement, Paris, Macula, 1998, p. 225-54 ; Hans-Michael Schäfer, Die Kulturwissenschaftliche Bibliothek Warburg: Geschichte und Persönlichkeiten der Bibliothek Warburg, Berlin, Logos Verlag, 2005.

4. Pour une mise en œuvre proprement philologique de la méthode warburgienne, voir Dieter Wuttke,

« Kunstgeschichte und Philologie ", Dazwischen : Kulturwissenschaft auf Warburgs Spuren, BadenBaden, Valentin Koerner, 1996, vol. 1, p. 3-64.

5. Giorgio Agamben, « Aby Warburg et la science sans nom », trad. Marco dell'Omodarme, Image et mémoire, écrits sur l'image, la danse et le cinéma, Paris, Desclée de Brouwer, 2004, p. 9-36.

6. Agamben, « Science sans nom », p. 12.

7. Cité in Ernst Gombrich, Aby Warburg: an intellectual biography, London, Warburg Institute, 1970, p. 26 :

"Philosophie bekomme ich in dem Colleg von Usener genug. Professor Justi, den Haupt-Mann für Kunstgeschichte, werde ich im nächsten Semester hören, nachdem ich mich genauer mit eigentlich philologischen Studien beschäftigt habe.»

8. Gombrich, Aby Warbug, 28-30; Roland Kany, Mnemosyne als Programm. Geschichte, Erinnerung und die Andacht zum Unbedeutenden im Werk von Usener, Warburg und Benjamin, Tübingen, Niemeyer, 1987, p. 67-113.

9. Hermann Usener, "Philologie und Geschichtswissenschaft (1882) », Vorträge und Aufsätze, Leipzig und Berlin, B. G. Teubner, 1907, p. 1-36.

10. Johannes Rössler, Poetik der Kunstgeschichte. Anton Springer, Carl Justi und die ästhetische Konzeption der deutschen Kunstwissenschaft, Berlin, Akademie Verlag, 2009.

11. Carl Justi, Die ästhetischen Elemente in der platonischen Philosophie: ein historisch-philosophischer Versuch (1859), Marburg, N. G. Elwert, 1860.

12. Karlheinz Stierle, "Altertumswissenschaftliche Hermeneutik und die Entstehung der Neuphilologie ", Philologie und Hermeneutik um 19. Jahrhundert- Zur Geschichte und Methodologie der Geisteswissenschaften, éds. Hellmut Flashar, Karlfried Gründer, Axel Horstmann, Göttingen, Vandenhoeck \& Ruprecht, 1979, p. 260-88. 
13. Anna Guillemin, «The Style of Linguistics: Aby Warburg, Karl Voßler, and Hermann Osthoff », Journal of the History of Ideas 69/4 (October 2008), p. 605-26.

14. Voir le catalogue numérisé de l'archive de l'institut Warburg : http:// calmview.warburg.sas.ac.uk/calmview/Record.aspx?

src $=$ CalmView.Catalog\&id=WIA+GC\%2f19395\&pos $=16$.

15. Karl Voßler, Positivismus und Idealismus in der Sprachwissenschaft. Eine sprach-philosophische Untersuchung, Heidelberg, Walther Verlag, 1904.

16. Paolo D'Angelo, « Aby Warburg e Benedetto Croce ", Aby Warburg e la cultura italiana, éds. Claudia Cieri Via and Micol Forti, Roma e Milano, Sapienza Università di Roma, 2009, p. 15-26.

17. Jesinghausen-Lauster, Suche nach der symbolischen Form, 257-270 ; Dieter Wuttke éd., Kosmopolis der Wissenschaft. E. R. Curtius und das Warburg Institute. Briefe und andere Dokumente, Baden-Baden, Koerner Verlag, 1989; Dieter Wuttke, « Ernst Robert Curtius und Aby M. Warburg ", Dazwischen vol. 2, p. 667-688.

18. Guillemin, « Style of linguistics », p. 614-5.

19. Avec Karl Brugmann, Ossthoff édita la revue Morphologische Untersuchungen auf dem Gebiete der Indogermanischen Sprachen, Leipzig, 1878-1890.

20. R. Meringer, W. Meyer Lübke, J. J. Mikkoa, R. Much et M. Murko, Wörter und Sachen. Kulturhistorische Zeitschrift für Sprach- und Sachforschung, vol. 1, Heidelberg, Carl Winters Universitätsbuchhandlung, 1909.

21. Matthew Rampley, « From symbol to allegory: Aby Warburg's theory of art ", The Art Bulletin LXXIX (March 1997), p. 46-7 ; Aby Warbrug, "Schlussitzung der Burckhardt Uebung », Werke in einem Band, Martin Treml, Sigrid Weigel und Pedita Ladwig Hrsg., Frankfurt am Main, Suhrkamp Verlag, 2010, p. 695-9.

22. James I. Porter, Nietzsche and the philology of the future, Stanford: Stanford University Press, 2000 ; Christian Benne, Nietzsche und die historisch-kritische Philologie, Berlin, Walter de Gruyter, 2005.

23. Karlfried Gründer éd., Der Streit um Nietzsches "Geburt der Tragödie" : die Schriften von E. Rohde, R. Wagner, U. v. Wilamowitz-Möllendorff, Hildesheim, Olms, 1989 ; Ingo Gildenhard et Martin Ruehl éds., Out of Arcadia : Classics and Politics in Germany in the Age of Burckhardt, Nietzsche and Wilamowitz, London, Institute of classical studies, School of advanced study, University of London, 2003.

24. Friedrich Nietzsche, "Wir Philologen », Werke in drei Bänden, éd. Karl Schlechta, München \& Wien, Hanser, 1999 (1956), vol. 3, p. 323-32 ; Friedrich Nietzsche, Sur la personnalité d'Homère ; Nous autres philologues, trad. Guy Fillion, Nantes, Le passeur, 1992.

25. Aby Warburg, " Ein unveröffentlicher Brief Warburg an Ulrich v. Wilamowitz-Moellendorff », in Jesinghausen-Lauster, Suche nach der symbolischen Form, p. 311-3.

26. Voir aussi Stefan Rieger, "Richard Semon und/oder Aby Warburg. Mneme und/oder Mnemosyne », Deutsche Vierteljahren Schrift für Literaturwissenschaft und Geistesgeschichte, Stuttgart und Weimar, 1998, p. 245-63.

27. Raymond Aron, La philosophie critique de l'histoire. Essai sur une théorie allemande de l'histoire, Paris, J. Vrin, 1969.

28. Stierle, «Altertumswissenschaftliche Hermeneutik und die Entstehung der Neuphilologie ", p. 260-1.

29. Erwin Panofsky, "Der Begriff des Kunstwollens (1920) ", Aufsätze zu Grundfragen der Kunstwissenschaft, eds. Hariolf Oberer and Egon Verhezen, Berlin, B. Hessling, 1964, p. 44.

30. Michel Foucault, Les Mots et les choses. Une archéologie des sciences humaines, Paris, Gallimard, 1966, p. 292-318.

31. Aby Warburg, « Nimfa Fiorentina (1900) », Werke, p. 198-209.

32. Andreas Jolles, Einfache Formen. Legende, Sage, Mythe, Rätsel, Spruch, Kasus, Memorabile, Märchen, Witz. Halle (Saale): Forschungsinstitut für Neuere Philologie Leipzig, 1930 ; André Jolles, Formes 
simples, trad. Marie Buquet, Paris, Seuil, 1972. Voir aussi Jesinghausen-Lauster, « Das System eines uersprünglichen Medien Kosmos bei Andre Jolles ", Die Suche nach der symbolischen Form, 246-56.

33. Sigrid Weigel a déjà souligné l'importance de ce terme. Sigrid Weigel, « Zur Archäologie von Aby Warburgs Bilderatlas Mnemosyne ", Die Aktualität des Archäologischen in Wissenschaft, Medien und Künsten, éd. Knut Ebeling und Stefan Altekamp, Frankfurt am Main, Fischer Taschenbuch Verlag, 2004, p. 199-200.

34. Voir Anne-Marie Christin, L'invention de la figure, Paris, Flammarion 2001 ; Catherine Malabou, Plasticité, Paris, Léo Scheer, 2000.

35. Warburg, « Nimfa Fiorentina », p. 203 ; Gombrich, Aby Warburg, p. 113.

36. Warburg s'est confronté à la question du conflit entre idéalisme et réalisme dans d'autres écrits, voir, par exemple Aby Warburg, "Florentinisnce Wirklichkeit und antikisirender Idealismus (1901)», Werke, p. 211-31.

37. Étienne Gilson, «L'être poiétique », Introduction aux arts du beau, Paris, Vrin, 1998, p. 101-120 ; René Passeron éd., La poiétique comme science et comme philosophie de la création, Paris, Éd. Poïesis, 1991.

38. Sur l'arché, voir Aristote, Métaphysique, livres A à E, trad. Bernard Sichère, Paris, Pocket Agora, 2007, p. 137-8 (1013a) ; Aristote, La Physique, trad. A. Stevens, Paris, J. Vrin, 1999, 67-96 (184a10-192b7).

39. Erwin Panofsky, Meaning in the visual arts, Garden City, NY, Doubleday Anchor Books: 1955, p. 38-40; Erwin Panogsky, Essais d'iconologie: Les thèmes humanistes dans l'art de la renaissance, I, C. Herbette et B. Teyssèdre, Paris, Gallimard, 1967, p. 29-31.

40. Voir Félix Ravaisson, De l'habitude (1838), Métaphysique et morale (1893), Paris: Puf, 1999 ; Adi Efal,

« Habitude against itself: Redefining the 'symbol' in turn-of-the-century French visual symbolist Discourse », Æ-Canadian Aesthetics Journal 13 (Summer 2007), http://www.uqtr.uquebec.ca/AE/ Vol_13/libre/efal2.htm.

41. Sur la relation entre philologie et scepticisme voir Christian Benne, « Philologie und Skepsis ", Was ist eine philologische Frage?, Jürgen Paul Schwindt ed., Frankfurt am Main, Suhrkamp Tachenbuch, 2009, p. 192-210; Thomas Steinfeld, « Skepsis. Über August Böckh, die Wissenschaft der unendlichen Approximation und das Glück der mangelden Vollendung», Was ist eine philologische Frage, p. 211-25.

42. James C. Morrison, "Vico's principle of verum is factum and the problem of historicism ", Journal of the History of Ideas 39/4 (Oct. - Dec., 1978), p. 579-95 ; Jean-Robert Armogathe, « Doute méthodique et morale cartésienne ", Kriterium. Revista de Filosofia 43106 (December 2002), p. 9-19, http://www.scielo.br/scielo.php?

script=sci_arttext\&pid=S0100-512X2002000200002\&lng=en\&nrm=iso .

43. Henri Bergson, La pensée et le mouvant : essais et conférences (1934), Paris, Presses Universitaires de France, 2009, p. 211.

44. Aby Warburg, "La Naissance de Vénus et le Printemps de Sandro Boticelli », Essais Florentins, trad. Sybille Muller, Paris, Éditions Klincksieck; 1990, p. 49 ; Aby Warburg, « Sandro Botticellis "Geburt der Venus" und Frühling" (1893) », Werke, p. 39: « In der vorliegenden Arbeit wird der Versuch gemacht, zum Vergleiche mit den bekannten mythologischen Bildern des Sandro Botticelli, der "Geburt der Venus" und de "Frühling" die entsprechenden Vorstellungen der gleichzeitigen kunsttheoretischen und poetischen Litteratur heranzuziehen um auf diese Weise das, was die Künstler des Quattrocento an der Antike "interessierte", klarzulegen. Es lässt sich nämlich hierbei Schritt bei Schritt verfolgen, wie die Künstler und deren Berater in "der Antike" ein gesteigerte äussere Bewegung verlangendes Vorbild sahen und sich an antike Vorbilder anlehnten, wenn es sich um Darstellung äusserlich bewegten Beiwerks- der Gewandung und der Harre- handelte. " 
45. Dans l'ex libris de Warburg lui-même (qui se trouve à la bibliothèque de l'institut Warburg à Londres), j'ai pu noter plusieurs exemplaires des écrits de Bergson (quelques-unes dans l'édition française mais la plupart dans la traduction allemande) et à des essais et livres sur l'œuvre de Bergson publiés autour de 1912-1913.

46. Sur la place de l'idée platonicienne dans l'histoire de la théorie de l'art, voir Erwin Panofsky, Idea. Contribution à l'histoire du concept de l'ancienne théorie de l'art (1924), trad. H. Joly, Paris, Gallimard, 1983 ; Adi Efal, « Panofsky's Idea and Auerbach's Figura: Two iconodulist philological experiments ", The Protocols of the History and Theory Department of the Bezalel Academy of Jerusalem 14 (October 2009), http://bezalel.secured.co.il/zope/home/en/1252746792 .

47. Sur la relation entre figuration et mouvement voir Gottfried Boehm, Gabriele Brandtstetter et Achatz von Müller eds., Figur und Figuration : Studien zu Wahrnehmung und Wissen, Munich, Fink Verlag, 2007.

48. Gilles Deleuze, Francis Bacon. Logique de la sensation, vol. I, Paris, La Vue le Texte, 1981, p. 19.

49. Richard Terdiman, Present Past : Modernity and the Memory Crisis, Ithaca, Cornell University Press, 1993 ; Mathew Rampley, The remembrance of things past. On Aby M. Warburg and Walter Benjamin, Wiesbaden, Harrasowitz Verlag, 2000.

50. Daniel Strassberg, Das poietische Subjekt : Giambattista Vicos Wissenschaft vom Singulären, München, Wilhelm Fink Verlag, 2007.

51. Sur philologie et mobilité générique voir Bernard Cerquiglini, Éloge de la variante : Histoire critique de la philologie, Paris, Seuil, 1989.

52. Georges Didi-Huberman, L'image survivante. Histoire de l'art et temps des fantômes selon Aby Warburg, Paris, Éditions de Minuit, 2002, p. 82-96.

53. Voir Erwin Panofsky, « L'histoire de l'art est une discipline humaniste», L' CEuvre d'art et ses significations, essais sur les arts visuels, trad. Marthe et Bernard Teyssèdre, Paris, Gallimard, 1969, 27-52; Dieter Wuttke, « Humanismus als integrative Kraft », Dazwischen, p. 389-454.

54. Pour un manifeste radical de la philologie anti-historiciste, voir Servais Étienne, Défense de la Philologie, Liège et Paris, Droz, 1933.

55. Erich Auerbach, Figura, trad. Diane Meur, Paris, Macula, 2003 ; Erich Auerbach, « Figura (1938) ", Gesammelte Aufsätze zur romanischen Philologie, Bern, Francke, 1967, p. 55-92.

56. Pierre Fontanier, Les figures du discours (1827), avec une introduction de Gérard Genette, Paris, Flammarion, 1968 ; Gérard Genette, « Figures », Figures I, Paris, Éditions du Seuil, 1966, p. 205-22.

57. Auerbach, Figura, p. 33-56 ; Auerbach, Figura, p. 65-74 (Version allemande). Voir aussi Karlfried Gründer, Figur und Geschichte : Johann Georg Hamanns "Biblische Betrachtungen" als Ansatz einer Geschichtsphilosophie, Freiburg et Munich, Albert, 1958.

58. Auerbach, Figura, p. 63-4: «L'interprétation figurative établit, entre deux événements ou deux personnages, une relation dans laquelle l'un des deux ne signifie pas seulement ce qu'il est mais est aussi le signe annonciateur de l'autre, qui l'englobe ou l'accomplit. » Et p. 69: « La prophétie figurative contient l'interprétation d'un événement intra- mondain par un autre ; le premier est le signe annonciateur du second, qui l'accomplit. Sans doute l'un et l'autre restent-ils des faits intra-historiques ; mais tous deux, dans cette perspective, ont cependant quelque chose de transitoire et d'incomplet ; chacun renvoie à l'autre (...). »

59. Martin Treml, «Auerbachs Imaginäre jüdische Orte », in Erich Auerbach : Geschichte und Aktualität eines europäischen Philologen, eds. Martin Treml et Karlheinz Barck, Berlin, Akademie Verlag, 2007, p. 246-51.

60. Marc de Launay, " postface », in Auerbach, Figura, p. 100-1.

61. Wuttke, Dazwischen, p. 309 ; Salvatore Settis, "Pathos et Ethos, Morfologia et fonzione ", Pathosformeln, retorica del gesto e rappresentazione : ripensando Aby Warburg, éd. Fabriozi Serra, Pisa, Istituti Editoriali e Poligrafici Internazionali, 2006, p. 23-34.

62. Erich Auerbach, Literatursprache und Publikum in der lateinischen Spätantike und im Mittelalter, Bern, Francke, 1958, p. 10 ; Erich Auerbach, Literary Language and its public in late Latin antiquity 
and in the middle ages, New Jersey, Princeton University Press, 1965, p. 7. Voir aussi Ernst Robert Curtius, Französischer Geist im 20. Jahrhundert, Tübingen und Basel, Francke, 1952.

\section{INDEX}

Mots-clés : expérimentation philologique, figurale, Neuphilologie, morphologie, supplétion, Lebensphilosophie

\section{AUTEUR}

\section{ADI EFAL}

Actuellement, elle est engagée dans un projet de recherche autour du concept de l'habitus, entre Ravaisson et Aristote, à l'institut de Thomas de l'université de Cologne, soutenue par la fondation Gerda Henkel. Publications récentes et à paraître : (Prévue) ‘Gravity of a figure,' Journal of visual arts practice, dir. Mick Finch et Jane Lee, 2013; (Prévue) ‘Der Erhalt poietische Dinge als Aufgabe der Kunstgeschichte,' en Gegenwart des Fetischs. Dingkonjunktur und Fetischbegriff in der Diskussion, dir. Christine Blaettler et Falko Schmieder, Turia \& Kant, 2013; (Prévue) 'Art History with less conditions of possibility: Following Bergson's 'le possible et le reel', en Bergson and the art of immanence, dir. Charlotte de Mille et John Mullarkey, Edinburgh University Press, 2013; 'Philology and the history of art,' The Making of the Humanities Volume II : From Early Modern to Modern Disciplines, dir. Rens Bod, Jaap Maat and Thijs Weststeijn, Amsterdam University Press, 2013, 263-299; 'Alois Riegl's concept of age-value and the challenge of historicism in the history of art,' Art History Supplement 1/5 (December 2011),https://sites.google.com/a/arths.org.uk/arths/ about/arthsa/issue5?pli=1; 'Reality as the cause of art: Alois Riegl and neo-kantian realism,' Journal of Art Historiography 3 (December 2010), http://arthistoriography.files.wordpress.com/ 2011/02/media_183171_en.pdf. 